This item was submitted to Loughborough's Research Repository by the author.

Items in Figshare are protected by copyright, with all rights reserved, unless otherwise indicated.

\title{
Atomistic surface erosion and thin film growth modelled over realistic time scales
}

PLEASE CITE THE PUBLISHED VERSION

http://dx.doi.org/10.1063/1.3657436

PUBLISHER

(c) American Institute of Physics

VERSION

VoR (Version of Record)

LICENCE

CC BY-NC-ND 4.0

\section{REPOSITORY RECORD}

Scott, Chris, Sabrina Blackwell, Louis J. Vernon, Steven D. Kenny, Michael Walls, and Roger Smith. 2019. "Atomistic Surface Erosion and Thin Film Growth Modelled over Realistic Time Scales". figshare. https://hdl.handle.net/2134/9364. 
This item was submitted to Loughborough's Institutional Repository (https://dspace.lboro.ac.uk/) by the author and is made available under the following Creative Commons Licence conditions.

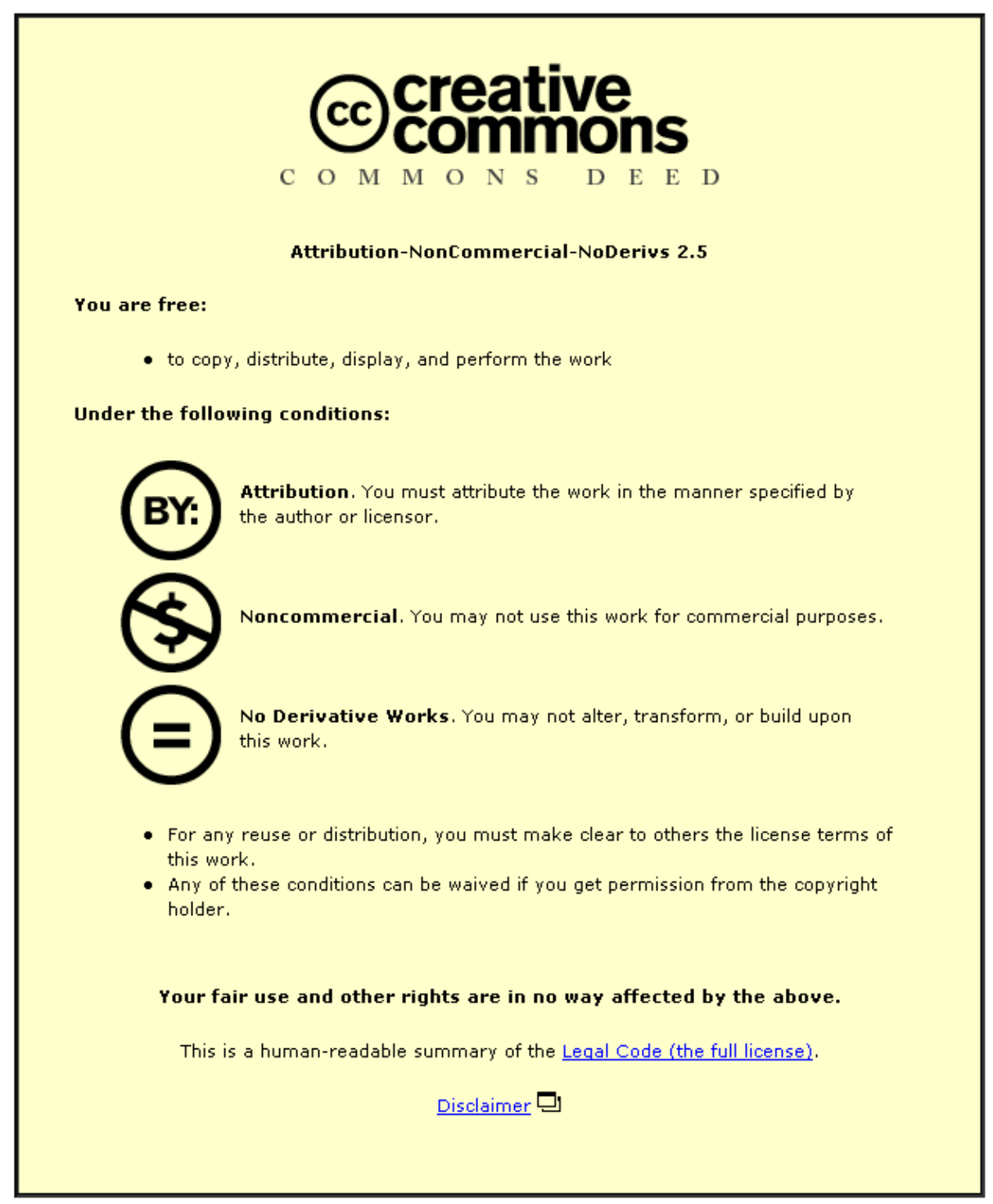

For the full text of this licence, please go to: http://creativecommons.org/licenses/by-nc-nd/2.5/ 


\title{
Atomistic surface erosion and thin film growth modelled over realistic time scales
}

\author{
Chris Scott, ${ }^{1}$ Sabrina Blackwell, ${ }^{1}$ Louis Vernon, ${ }^{1, \text { a) }}$ Steven Kenny, ${ }^{1}$ \\ Michael Walls, ${ }^{2}$ and Roger Smith ${ }^{1, b)}$ \\ ${ }^{1}$ Department of Mathematical Sciences, Loughborough University, Loughborough, LE11 3TU, \\ United Kingdom \\ ${ }^{2}$ Department of Electrical Engineering, Loughborough University, Loughborough, LE11 3TU, United Kingdom
}

(Received 22 September 2011; accepted 10 October 2011; published online 3 November 2011)

\begin{abstract}
We present results of atomistic modelling of surface growth and sputtering using a multi-time scale molecular dynamics-on-the-fly kinetic Monte Carlo scheme which allows simulations to be carried out over realistic experimental times. The method uses molecular dynamics to model the fast processes and then calculates the diffusion barriers for the slow processes on-the-fly, without any preconceptions about what transitions might occur. The method is applied to the growth of metal and oxide materials at impact energies typical for both vapour deposition and magnetron sputtering. The method can be used to explain growth processes, such as the filling of vacancies and the formation of stacking faults. By tuning the variable experimental parameters on the computer, a parameter set for optimum crystalline growth can be determined. The method can also be used to model sputtering where the particle interactions with the surface occur at a higher energy. It is shown how a steady state can arise in which interstitial clusters are continuously being formed below the surface during an atom impact event which also recombine or diffuse to the surface between impact events. For fcc metals the near surface region remains basically crystalline during the erosion process with a pitted topography which soon attains a steady state roughness. (C) 2011 American Institute of Physics. [doi:10.1063/1.3657436]
\end{abstract}

\section{INTRODUCTION}

The interaction of energetic ions (and neutrals) with surfaces has a plethora of important high technology applications. Thin film deposition is used to manufacture a range of thin films used in applications such as low emissivity coatings on glass, integrated circuits, light-emitting diode and organic light-emitting diode displays, electrochromics, optical coatings, and solar panels. A variety of techniques are used to deposit thin films on an industrial scale. The choice of technique is determined by the morphology required in the specific application and cost. The morphology is determined by the energy of deposition which varies according to the technique used. For example, the deposition of thin films using thermal and electron beam evaporation occurs at energies $<1 \mathrm{eV}$. Higher energies can be imparted to the growing film by ion assist using an ion source with energies $>100 \mathrm{eV}$. Magnetron sputtering is another industrial scale technology that employs a plasma and typically deposits atoms in the energy range of $10 \mathrm{eV}-50 \mathrm{eV}$. Conversely, low energy ( $>100 \mathrm{eV}$ ) ion beams are used to etch surfaces. For example, ion etching is a commonly used tool in the manufacture of semiconductor devices.

Both thin film deposition and ion etching occur at the atomic level over time scales that have been traditionally very

\footnotetext{
${ }^{a)}$ Present address: Los Alamos National Laboratory, Los Alamos, New Mexico 18745, USA.

b) Author to whom correspondence should be addressed. Electronic mail: R.Smith@lboro.ac.uk.
}

difficult to access by molecular dynamics (MD). This paper introduces new computational techniques that allow the atomic scale interactions to be modelled on realistic time scales. This new modelling approach reveals mechanisms at work which explain experimental observations that are complex and non-intuitive.

MD is an extremely powerful tool for investigation of many phenomena from low energy chemical reactions to high energy radiation effects. It can be used with both classical empirical potentials to model the atomic interactions or through the direct use of $a b$ initio methods to determine the interatomic forces. Since the pioneering work of Alder and Wainwright ${ }^{1}$ investigating phase transitions in a hard sphere system and that of Vineyard's group at Brookhaven ${ }^{2}$ investigating radiation effects, the technique has found ever increasing application to more and more complex systems. As computers have become more powerful, it has been possible to study larger and larger systems modelled by empirical potentials through the use of parallel processing and spatial decomposition. In addition linking to continuum models has meant that it has been possible for many applications to bridge the gap between microscopic length scales and length scales accessible by observation in laboratory experiment. ${ }^{3}$

The technique has, however, suffered from a considerable drawback. The method relies on the numerical integration of Newton's equations of motion for the interacting particles and because of the nature of forces and distances involved to obtain an accurate numerical result, the integration time step cannot be much greater than the order of $1 \times 10^{-15} \mathrm{~s}$, i.e., 
1 femtosecond (fs). Even with an efficient integrator, such as the Verlet algorithm, ${ }^{4}$ the forces on the atoms have to be evaluated at least once per time step so in order to simulate $1 \mathrm{~s}$ of real time $10^{15}$ force evaluations for each atom in the system, have to be performed. This has usually meant that simulations have been limited to time scales at the most of the order of nanoseconds to microseconds. This is fine, for example, in the investigation of the ballistic phase of a radiation event caused by a nuclear reaction or that caused by a single particle impact on a surface, where the ballistic phase only lasts a few picoseconds, but the method fails when dose effects are to be considered. For example in magnetron sputtering for a typical deposition rate is one monolayer per second equivalent to $\approx 200$ separate deposition events in $1 \mathrm{~s}$ on a surface area of $\approx 2.5 \mathrm{~nm} \times 2.5 \mathrm{~nm}$, a typical size that can be handled computationally. Thus, such a time scale is clearly outside the region of computational accessibility by MD alone.

This problem has been recognised for many years and a number of groups have developed clever long time scale methods to try to address the problem..$^{5-13}$ The key point that allows the issue to be addressed is that after an initial ballistic collisional event, the dynamical system then changes its state only very slowly through the occurrence of rare events.

The issue is highlighted by the example shown in Fig. 1. In Fig. 1(a), particle moves in the $x-y$ plane under the influence of a force field derived from the gradient of the potential function $V=\sin (2 x) \cos (x y)$. The dark red regions are the minima of the potential function and the lighter regions the maxima. Four saddle points are marked with an "x," surrounding the basin, A, associated with the local minimum. Initially the particle is given a certain velocity in a random direction and remains initially trapped in the basin $\mathbf{A}$ before a transition occurs jumping into the next basin at B. As the dynamics continues the particle remains mainly trapped in the basin around a minimum, occasionally making the jump between basins A, B, C, D, and E. Thus, the long term dynamics is governed not by the motion in an actual basin but by the heights of the saddle points between them and the number of attempts to cross. This is the key to linking the short time and long time behaviour. This aspect of the problem is the basis of transition state theory, originally developed for studying chemical reactions ${ }^{14,15}$ but equally applicable to the cases described here.

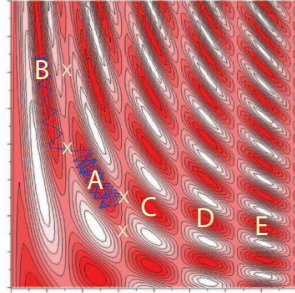

(a)

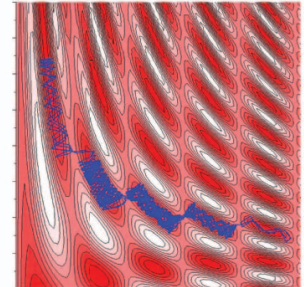

(b)
FIG. 1. The track (blue line) of a particle moving in the potential field $V$ $=\sin (2 x) \cos (x y)$ with the different basins of attraction in which the particle remains temporarily trapped marked by A, B, C, D, and E. The crosses mark the saddle points surrounding the initial basin A. The particle initially starts in basin $\mathrm{A}$ before jumping into B shown in (a). The right hand figure (b) shows the system at a later time.
In a real system where the potential energy surface is more complex than that illustrated by the simple example, it is very difficult to know in advance all the saddle points surrounding a local minimum where a dynamical system is temporarily trapped. Traditional kinetic Monte Carlo (KMC) methods, developed to access long time scales have generally been lattice based involving evolving the system from a pre-defined events table. However, Henkelman and Jønnson ${ }^{16}$ have shown that it is also possible to determine the transitions (saddle points and their heights) between basins "on-the-fly" (otf-KMC), i.e., without any pre-knowledge of what transitions were likely to occur. By doing this unexpected concerted events were picked up that would be otherwise missed.

In this paper we combine traditional MD with otf-KMC in a multi-time scale approach to study two important problems; first, that of modelling thin film growth over realistic experimental time scales. This could be either evaporation deposition or deposition by magnetron sputtering, depending on the impact energy of the arriving species. As the energy of the arriving species increases further, a net amount of material is removed rather than added, causing surface erosion and sputtering. This is the second problem investigated here. Many previous MD studies of thin film growth have been undertaken at abnormally high deposition rates although Sprague and Voter, ${ }^{17}$ by using temperature accelerated dynamics, have shown that surface growth occurred differently when long time scale effects are considered. Similarly, MD sputtering studies from the pioneering work of Harrison in the late 1960 s and early 1970 s (Ref. 18) have really only considered individual impact events rather than dose effects so that whereas angular ejection patterns from crystals, Wehner spots, ${ }^{19}$ and ejected ion energy distributions could be accurately modelled, predicted sputtering yields have generally never been in very close agreement with experiment due in part to the damage inflicted on a crystal by successive ion impacts. Thus multi-time scale methods will be used (1) to identify the mechanisms by which thin films grow; (2) to provide information to experimentalists involved with magnetron sputtering to help predict the optimum conditions for growth; and (3) to examine dose effects in sputtering and surface erosion and the damage mechanisms.

\section{METHODOLOGY}

The system dynamics both for deposition and sputtering is divided into two time regions, a short region lasting a few picoseconds during which the ballistic processes take place and the system is studied by MD, followed by an otf-KMC stage that occurs between impact events. The key point about the otf-KMC stage is the use of the harmonic approximation and transition state theory to determine the time scales for the rare events that drive the diffusion process between deposition events. Recent work, using some simple cases, to test the numerical accuracy of the assumption has shown that diffusion coefficients calculated for a simple system using both MD and transition state theory are in good agreement, provided the temperature is not so high that anharmonic effects are important. ${ }^{20}$ In our case the simulations are carried out at $350 \mathrm{~K}$ so anharmonic effects are unlikely to be dominant. The 
way in which the two methodologies are linked is described in detail below.

\section{A. Molecular dynamics}

During the MD stage, the bottom layer of the lattice was fixed and a thermostat ${ }^{21}$ was attached to the next two layers to remove any excess energy from the system caused by a particle impact. For deposition, single atoms or randomly oriented ad-units were deposited normally to the surface, either with a fixed energy $E_{d}$ or with energies selected from a normal distribution with a certain standard deviation, typically a tenth of $E_{d}$. Ar atoms are also allowed to strike the substrate if required. Ar is present naturally in a magnetron sputtering system and can also be introduced as an ion assist process used for the densification of thin films produced by evaporation. Experimentally, sputtering is also usually carried out by bombardment with Ar ion beams. The substrate was always heated to a required temperature before a deposition event, typically $350 \mathrm{~K}$ and MD ran until the lattice returned to this temperature after the energetic impact and the defects had stabilised, after which time the lattice was relaxed. This typically took 3-20 ps, depending on deposition energy used (longer for the higher energy sputtering simulations). After relaxation, the KMC phase begins.

We studied deposition of $\mathrm{TiO}_{2}$, a complex oxide, described by a variable charge potential ${ }^{22,23}$ and also deposition on a simple close-packed metal (Ag). For the sputtering simulations, an Au substrate was chosen as it is known to have a high sputtering yield. The metal force fields were modelled using an embedded-atom type potential, ${ }^{24}$ the Ar-metal interactions using a screened Coulomb potential ${ }^{25}$ and the Ar-Ar interactions by a Lennard-Jones potential. ${ }^{26}$ Where necessary, the potentials were splined to a screened Coulomb potential for close particle separation. At the start of the simulation a rectangular block of a perfect crystal was chosen with its surface in the $y$ direction. Periodic boundary conditions were imposed in the $x-z$ plane, and the impacting particles placed at a sufficient distance above the surface.

\section{B. On-the-fly kinetic Monte Carlo}

KMC (Ref. 27) involves pre-definition of all available transitions. This is useful for simple and symmetric crystal structures, but traditional KMC cannot perform accurately as systems become more complex. When dealing with highly defective systems, it becomes almost impossible to pre-define all the transitions. Otf-KMC (Ref. 16) aims to rectify this issue by calculating transitions on-the-fly. Our otf-KMC algorithm involves four fundamental steps, described below:

1. Identify of all the defects by comparison to a perfect bulk lattice. This produces a sub-system where defects and their neighboring atoms are included. It is only atoms in this sub-system that are included in the search for a transition. In theory, one could choose all particles in the original lattice which is what temperature accelerated dynamics (TAD) (Ref. 6) does, but by restricting the transition searches to the defective regions and by using a direct search for the saddle points rather than dy- namics to find the transitions (as in Fig. 1), considerable computing time can be saved.

2. Search for all possible transitions. Locate saddle points using the relaxation and translation method, ${ }^{28}$ and then use the climbing image nudged elastic band method ${ }^{29}$ to determine barrier heights more accurately once the transition has been found. Typically, 200 searches are carried out.

3. Calculate transition rates of every unique transition found. Use the Arrhenius equation escape frequency $=v \exp \left(-E_{b} / k_{B} T\right)$, where $v$ is the transition pre-factor, $E_{b}$ is the transition barrier, $k_{B}$ is the Boltzmann constant, and $T$ is the temperature. The pre-factor $v$ can be calculated for each transition using the Vineyard method ${ }^{30}$ but since the transition rate depends much more strongly on $E_{b}$ than $v$ which was found to vary by only a small amount, $v$ was calculated only once and a typical value chosen. A good value for the pre-factor was found to be $\approx 10^{13} \mathrm{~s}^{-1}$. It is also possible at this stage to block transitions from the list, for example, if a transition involves a process that involves no net diffusion, such as an atomic vibration between equivalent sites. This can be done either from a recognition of the local environment or by the cruder method of ignoring all transitions with especially low energy barriers.

4. Carry out transition searches in parallel together with a deposition event. These are done typically on between 24 and $48 \mathrm{cpu}$ cores. Either a transition or deposition event is chosen from a roulette algorithm and is used to evolve the system in time. With modern computers it is very straightforward to write a script that distributes these calculations to different processors and is an essential step that makes the method computationally feasible.

\section{APPLICATIONS}

\section{A. Deposition of silver atoms on silver surfaces}

In this section we consider low energy deposition of silver atoms on the $\operatorname{Ag}\{111\}$ surface. We assume that the arriving atoms impact normally on the surface and we consider the effect of varying the deposition energy. Here, deposition energies of $1 \mathrm{eV}$ and $40 \mathrm{eV}$ only are considered as these may be typical, respectively, of a vapour deposition process and one involving magnetron sputtering. A deposition rate of ten monolayers per second is chosen in both cases. This is on the high side of what is used in practice. The modelling of deposition of atoms on crystal surfaces has been considered by many authors over the years either using KMC algorithms ${ }^{16}$ or MD (Refs. 17 and 31) but some of the basic mechanism by which growth occurs are still the subject of conjecture. Work involving TAD has also shown that growth patterns are substantially different if long time scale effects are properly considered. ${ }^{17}$

Recent experiments using low energy ion scattering to examine the first few layers grown by vapour deposition on $\{111\}$ surfaces have shown that growth does not always occur in a totally crystalline manner. ${ }^{32}$ The grown structure can contain both stacking faults and twin boundaries. Previous work has also shown that single atoms are very mobile over the sur- 


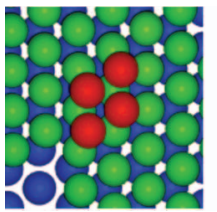

(a)

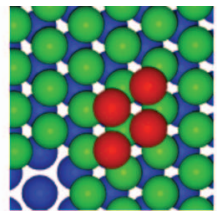

(b)

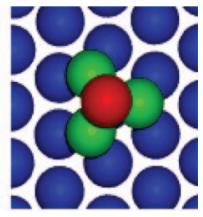

(c)
FIG. 2. Four-atom Ag clusters on the Ag $\{111\}$ surface. The bottom (blue) layer of atoms is labelled as the "A" site and the green layer the "B" site. (a) The C site position; (b) the A site position; and (c) the non-planar unit. The energy barrier for motion from the position shown in (a) to (b) is $0.40 \mathrm{eV}$ whilst the reverse barrier is $0.26 \mathrm{eV}$.

face but growth can still nucleate without the need of a seed point (such as a surface defect) to which the mobile atoms can stick. $^{33}$

We also find that both single $\mathrm{Ag}$ atoms and $\mathrm{Ag}$ dimers are very mobile on the surface with energy barriers for diffusion of $0.12 \mathrm{eV}$ and $0.22 \mathrm{eV}$, respectively. Surface trimers are also mobile and are found to diffuse as a unit with an energy barriers of $0.28 \mathrm{eV}$. The trimer can also rotate through $60^{\circ}$ with an energy barrier of $0.23 \mathrm{eV}$. Thus, to save time at the start of our simulations we start with a trimer as a unit on the surface. This trimer takes the form of an equilateral triangle. For low energy deposition, where the atoms arrive on the surface with an impact energy of $1 \mathrm{eV}$, the simulations then show that a deposited atom then diffuses over the surface until it joins a surface trimer producing a quadramer, Fig. 2. This quadramer can also diffuse with a value of $0.40 \mathrm{eV}$ from its most stable site and now the expected hop time is similar to the likelihood of another atom depositing in the neighboring region on the surface at room temperature. If a further deposited atom joins to produce a five-atom planar cluster then the barrier increases to $0.45 \mathrm{eV}$ and the hop time decreases from $1.9 \times 10^{6}$ times per second to $2.6 \times 10^{5}$ times per second and only moves rarely between deposition events. Detachment from the 5-atom cluster or diffusion of the cluster is also low probability events that are unlikely to occur before the first monolayer is deposited.

The quadramer is a planar unit which takes the form of a parallelogram and if we consider that the $\{111\}$ structure is stacked in the A, B, C form (in the usual notation) with the surface being the A plane, then the quadramer can sit in either the B or C position, Figs. 2(a) and 2(b), depending on where the trimer was situated when it was joined by a diffusing adatom. The energy barriers for diffusion of the quadramer are $0.40 \mathrm{eV}$ from the $\mathrm{B}$ to $\mathrm{C}$ position and $0.26 \mathrm{eV}$ for the reverse motion. Although the B position is a more stable site, the unit can move in either direction in the time during which a new adatom would arrive in a neighboring region of the surface. There is also a more mobile non-planar quadramer that consists of a surface trimer with an atom sitting out-of-plane above the centroid of the other three. This unit can move in a similar fashion to the surface trimer with similar barriers and can both rotate and diffuse. Such a unit is shown in Fig. 2(c). This unit remains intact on the flat surface but can decay in a concerted motion involving 3-4 atoms when it comes into contact with a corner on a step edge. The topmost atom moves to take the place of an atom in the trimer unit while that atom drops onto the step edge in the layer below.

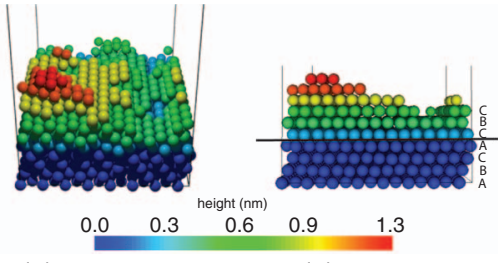

(a)

(b)

FIG. 3. Growth at $1 \mathrm{eV}$ after $0.29 \mathrm{~s}$ of real time with the addition of 675 atoms (equivalent to three complete monolayers), corresponding to a growth rate of ten monolayers per second. The original system contained 900 atoms. (a) Top view showing incomplete layers and (b) side view showing the stacking sequence.

It is also easy to see how both stacking faults and twinning can occur in the deposited film. Once a 5-atom cluster is formed on the surface it is usually static between deposition events and acts as a nucleation site for further arriving atoms. If it is pinned in the B layer position then the next layer will grow around it with the natural $\{111\}$ stacking. If it is pinned in the $\mathrm{C}$ position then the next layer contains a stacking fault. Thus, if two adjacent areas of the surface contain 5-atom clusters with one in the B position and another in the $\mathrm{C}$ position, the clusters will eventually grow and a twin boundary will form where they intersect. Figure 3 shows images of the growth at $1 \mathrm{eV}$ where a stacking fault has formed due to pinning a 5-atom cluster in the B position. One can see that at this energy, the growth is generally crystalline with stacking faults but although the sixth added layer is beginning to form, the second ad-layer is not yet complete.

As the growth continues many concerted mechanisms occur which could not have been predicted in advance in addition to the decay of the surface. One important example concerns the mechanism for vacancy filling. Vacancies in the grown layers are undesirable for optical applications and in Fig. 4 two important vacancy filling mechanisms are shown. The hole-filling often occurs not by single adatoms dropping into vacant hole, but by two-atom concerted motions. In Fig. 4(a), two atoms in the second added layermove as a unit on the edge of a second layer cluster, with one of the atoms dropping into an unfilled site in the first added layer. In Fig. 4(b), two atoms in the first added layer slide together to fill the vacancy.

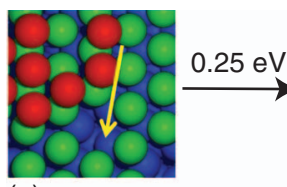

(a)

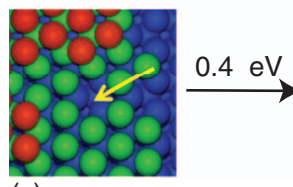

(c)

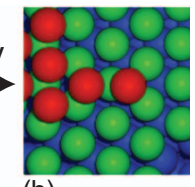

(b)

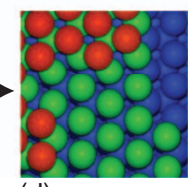

(d)
FIG. 4. Two-atom concerted motions showing different mechanisms by which vacancies in the growing layer are filled. 


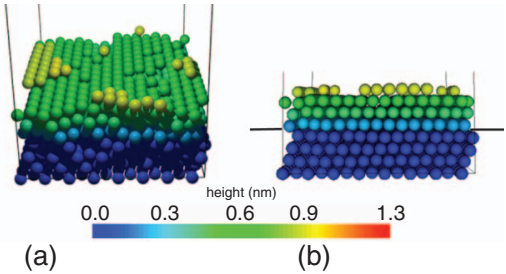

FIG. 5. Growth at $40 \mathrm{eV}$ after $0.27 \mathrm{~s}$ of real time with the addition of 675 atoms (equivalent to three complete monolayers), corresponding to a growth rate of ten monolayers per second. The original system contained 900 atoms. (a) Top view and (b) side view showing a perfect fcc stacking sequence.

As the energy of the depositing particles is increased to $40 \mathrm{eV}$, the quality of the crystalline growth increases. The lower energy of $1 \mathrm{eV}$ is insufficient to displace atoms and arriving particles can only diffuse over the surface. At $40 \mathrm{eV}$, particles can be displaced and atoms in the substrate can be exchanged with arriving atoms. The mixing and the added energy in the system leads to much smoother, more crystalline growth. Figures 3 and 5 allow a comparison between growth at $1 \mathrm{eV}$ compared to growth at $40 \mathrm{eV}$. Figure 3 shows that at $1 \mathrm{eV}$, we have six added layers of which only the first is complete and a stacking fault in the first added layer, whereas when the equivalent number of atoms is added at $40 \mathrm{eV}$, Fig. 5, there are nearly three complete layers in the perfect fcc structure. The larger energy leads to more complete layers without stacking faults. In fact, $40 \mathrm{eV}$ was found to be about the optimum energy for the best crystalline growth on for this system.

\section{B. Deposition on $\mathrm{TiO}_{2}$}

The method can also be applied to more complex systems. Here, we present some results for growth on $\mathrm{TiO}_{2}$, where the system is modelled by a variable charge potential, with a deposition rate of 0.5 monolayers per second used in the simulations, see Fig. 6. A variable charge potential is necessary since the potential needs to model on the one hand pure $\mathrm{Ti}$ in which the atoms are not charged, the oxygen dimer which is strongly bound and the various phases of the $\mathrm{TiO}_{2}$ which are ionic systems.
For the case of deposition on rutile, the $\{110\}$ surface was chosen as is the most stable. A mixture of different small molecular species was assumed to be incident on the surface, single $\mathrm{O}$ and $\mathrm{Ti}$ atoms, $\mathrm{TiO}$ and $\mathrm{TiO}_{2}$ units, and $\mathrm{O}_{2}$ dimers. For $\mathrm{TiO}_{2}$, not only the energy of the arriving species can be altered, but also the stoichiometry. Following a number of numerical experiments it was found that the most crystalline growth also occurs at deposition energies between 24 and $40 \mathrm{eV}$ and with an oxygen rich mixture for the arriving species. The stoichiometry details of the simulation are given in the caption to Fig. 6. Such a composition was found to be optimal but it is also necessary not to overdo the oxygen content as chemical etching of the surface can then occur. ${ }^{34}$

The rutile $\{110\}$ surface has been studied by a number of authors both experimentally and using ab initio methods. ${ }^{35-37}$ Key experimental findings are that the surface oxidises very easily and that as the surface is heated to remove the oxide layer, Ti interstitials are drawn out from the bulk. Our simulations indicate a similar growth mechanism whereby $\mathrm{Ti}$ interstitials are easy to implant at energies of a few tens of eV, whereas it is much more difficult for $\mathrm{O}$ atoms to penetrate and most deposit on the surface or reflect at these energies. Single $\mathrm{O}$ atoms are mobile on the surface between the raised $\mathrm{Ti}$ rows, (see Fig. 6(a)). The presence of oxygen on the surface lowers the energy barriers for Ti to escape and new ad-units are formed with the surface $\mathrm{O}$ atoms. This is a main mechanism by which new crystalline growth occurs. Figure 6(a) shows the growth from deposition at $1 \mathrm{eV}$. Notice that the new layers are incomplete, with both oxygen and titanium atoms missing. The low kinetic energy of the arriving atoms does not transfer enough energy into the lattice for much diffusion to occur, therefore leaving the layers incomplete with voids. With a $40 \mathrm{eV}$ deposition energy, shown in Fig. 6(b), it can been seen that the first two added layers have almost perfect crystalline growth.

\section{Sputtering of $\mathrm{Au}$}

When the energy of the bombarding species increases to a few hundred $\mathrm{eV}$ sputtering of the surface rather than growth

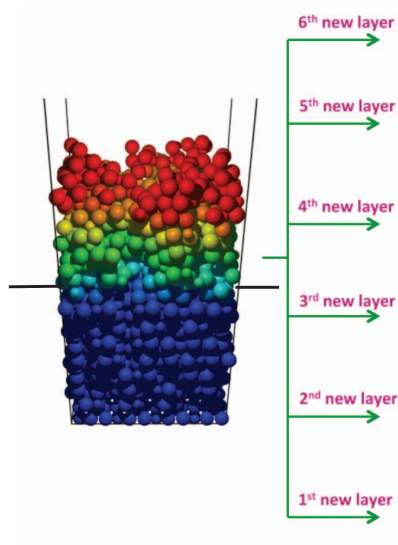

(a)

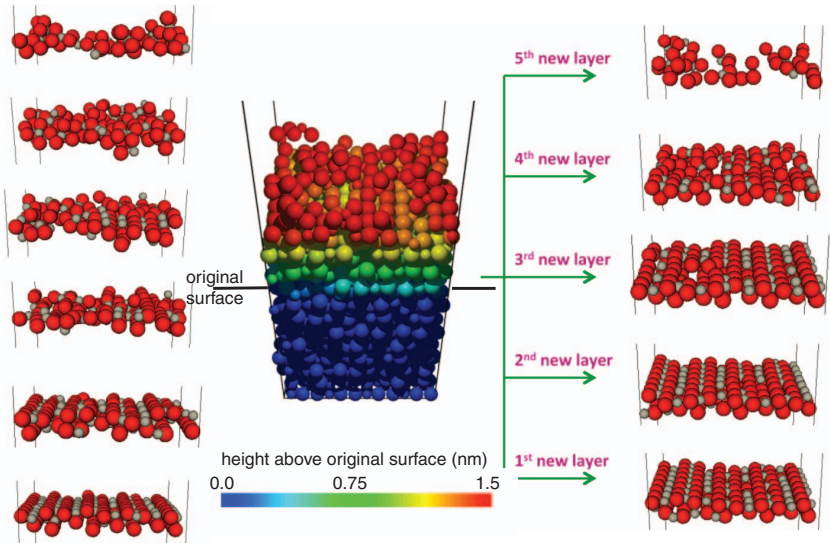

(b)

FIG. 6. Growth on rutile $\{110\}$ at (a) $1 \mathrm{eV}$ and (b) $40 \mathrm{eV}$. In both cases 768 atoms have been added to a substrate that contains 1536 atoms. The deposition rate was $\frac{1}{2}$ monolayers per second. The species arrive normally on the surface in the proportion $\mathrm{O} 10 \%$, $\mathrm{Ti} 10 \%$, TiO $30 \%$, $\mathrm{TiO}_{2} 20 \%$, and $\mathrm{O}_{2} 30 \%$. In the latter three cases the molecules are randomly orientated before impact. In (a) $5.3 \mathrm{~s}$ of real time is simulated in (b), $5.8 \mathrm{~s}$. 


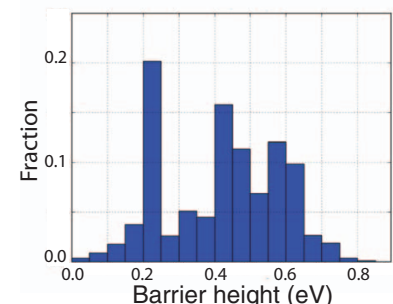

(a)

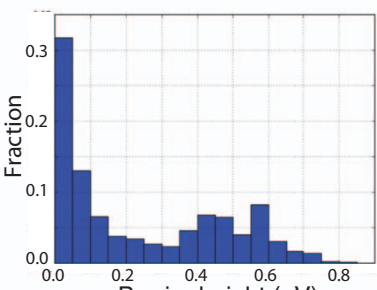

(b)

FIG. 7. The transition energy barriers for diffusion between impacts after bombardment of $\mathrm{Au}\{100\}$ at $500 \mathrm{eV}$ by (a) Au atoms and (b) Ar atoms. The large low energy tail in (b) is due to sub-surface Ar diffusion.

takes place. Here, we consider an Au crystal with a $\{100\}$ surface with atoms incident normally to the surface at room temperature. In one simulation, Ar atoms were chosen as the projectile, in the other Au atoms. An impact energy of $500 \mathrm{eV}$ was chosen for which an experimentally observed sputtering yield of about three atoms per incident ion for both Ar and Au bombardment has been reported. ${ }^{38}$ The substrate temperature was assumed to be $350 \mathrm{~K}$ for direct comparison with the deposition simulations. We perform the simulations in a similar manner to the growth calculations. A dose of $4 \times 10^{14}$ Ar and $7 \times 10^{14} \mathrm{Au}$ atoms $/ \mathrm{cm}^{2}$ is incident on a surface. The substrate in this case contains 8000 atoms with dimensions $4.1 \mathrm{~nm} \times 8.2 \mathrm{~nm} \times 4.1 \mathrm{~nm}$. A simulation corresponding to $0.20 \mathrm{~s}$ of real time was performed during which 66 successive Ar atoms impacted on the substrate and $117 \mathrm{Au}$ corresponding to a real time of $0.15 \mathrm{~s}$. Although all 8000 atoms in the system are included in the MD part of the simulation, when the defects and their surrounds are analysed this number is reduced to around 600-700 for inclusion in the search vector for transitions. Figure 7 shows the distribution of energy barriers determined for the saddle point searches. In Fig. 7(a), the barriers for Au self-bombardment are shown, in Fig. 7(b) the barriers for Ar bombardment. The distributions are similar apart from the larger number of low energy barriers in the Ar case. These correspond to the diffusion of Ar below the surface which makes the simulations somewhat slower than in the Au bombardment case. The Ar diffusion is also fastest when the Ar is clustered rather than isolated, Ar di-interstitials being especially mobile. The implanted Ar does not accumulate into large bubbles at these energies but either diffuses to the surface or is exposed as the surface erodes. On average about four Ar atoms remain in the system at any one time corresponding to $2.5 \times 10^{13}$ per $\mathrm{cm}^{2}$.

As the simulation progresses, adatoms are thrown up onto the surface, particles are ejected and a rough topography forms which eventually stabilises. A remarkable feature of the process is the amount of self-healing that occurs between ion impacts. Adatoms thrown up onto the surface attain crystalline form, such as an incomplete deposited layer in the growth simulations. Interstitial loops with up to 13 atoms form below the surface but eventually decay into smaller more mobile loops. Snapshots of the topography on the surface after various simulation times are shown in Fig. 8. Corresponding images of the surface atoms that lie above the height of the original surface and subsurface interstitial loops and implanted Ar are shown in Fig. 9. As the sur-

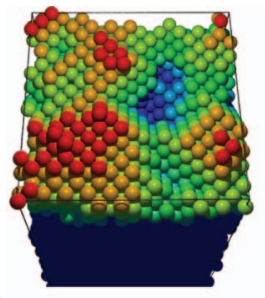

(a)

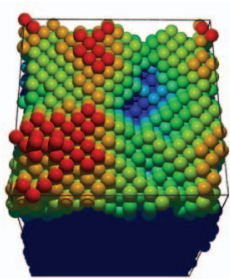

(b)

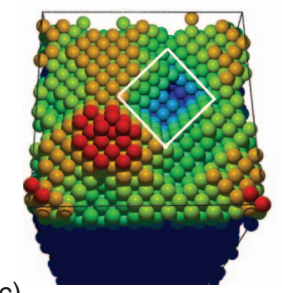

(c)

(d)

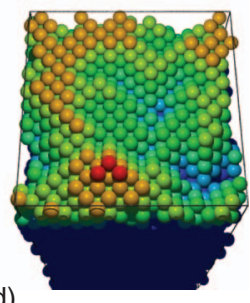

FIG. 8. The surface topography after various simulation times; (a) $0.085 \mathrm{~s}$ or $2.38 \times 10^{14} \mathrm{Ar} \mathrm{cm}^{-2}$; (b) $0.087 \mathrm{~s}$ or $2.56 \times 10^{14} \mathrm{Ar} \mathrm{cm}^{-2}$; (c) $0.010 \mathrm{~s}$ or $2.74 \times 10^{14} \mathrm{Ar} \mathrm{cm}^{-2}$; (d) $0.137 \mathrm{~s}$ or $3.69 \times 10^{14} \mathrm{Ar} \mathrm{cm}^{-2}$. The white diamond in (c) outlines a small facetted etch pit that has formed. Red spheres represent adatoms above the height of the original surface, green and blue atoms lie below the height of the original surface.

face erodes it becomes rougher but the roughness and sputtering yield eventually reach steady values. The calculated values of the sputtering yield per incident particle, for Ar and $\mathrm{Au}$ self-bombardment are 3.7 and 3.0, respectively. In addition to the distribution of atoms ejected per single impact looks very similar to those calculated for perfect crystal surfaces with its log-normal shape and a few high-yield events in the tail.

Figure 8 shows the development of a facetted crater which forms after $0.01 \mathrm{~s}$ and is marked by the diamond (a)

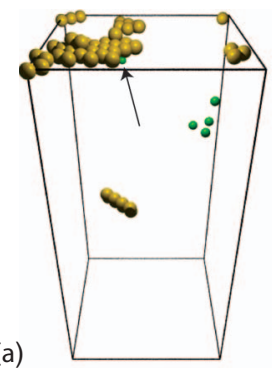

(c)

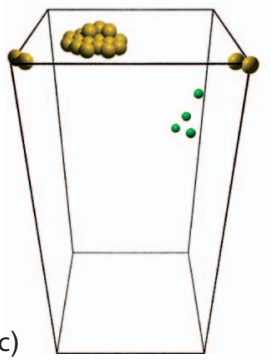

(b)

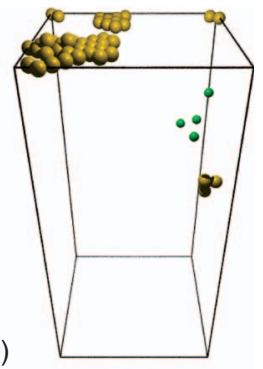

(d)

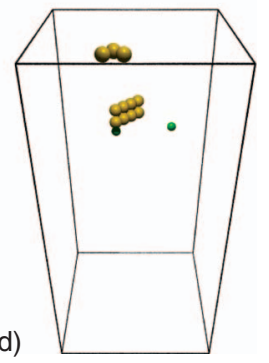

FIG. 9. Sub-surface interstitials and adatoms, above the height of the original surface, corresponding to the images shown in Fig. 8: (a) $0.085 \mathrm{~s}$; (b) $0.087 \mathrm{~s}$; (c) $0.010 \mathrm{~s}$; and (d) $0.137 \mathrm{~s}$. The small green spheres represent the implanted Ar atoms. One of these that diffuses to the surface is marked by the arrow in (a). 
shape in Fig. 8(c) but due to the continual ejection from and diffusion of ato in the substrate, the crater has disappeared as a recognisable entity by $0.137 \mathrm{~s}$ as shown in Fig. 8(d). Figure 9(a) shows a sub-surface interstitial loop of eight atoms, together with a 4-atom Ar cluster and an isolated $\mathrm{Ar}$ interstitial marked by the arrow. In Fig. 9(b), the isolated Ar atom has diffused to the surface $2 \mathrm{~ms}$ later and the interstitial loop has decayed to a three-atom cluster and totally disappears after a further $13 \mathrm{~ms}$, Fig. 9(c). Finally in Fig. 9(d), a new sub-surface 8 atom interstitial loop has formed and two further $\mathrm{Ar}$ atoms have been ejected. Thus, the erosion process continues with interstitial loops and craters continually forming and decaying as further Ar impacts on the surface.

The crystal healing process explains why the angular ejection patterns of sputtered material from a crystal observed experimentally show such sharp features. The prediction by Harrison and Garrison from single impact MD simulations on perfect crystal surfaces, ${ }^{18,39}$ that channelling and blocking by surface atoms is responsible for the patterns, also holds for higher experimental doses since the basic surface structure remains intact.

\section{CONCLUSIONS}

A multi-time scale dynamics method, has been applied to model atomistic deposition and erosion over realistic experimental time scales. For the application presented here, we have been able to explain the formation of stacking faults in thin film growth and identify concerted mechanisms that occur to fill surface vacancies during the growth process. We can predict the optimum energy for crystalline growth and in the case of a complex oxide, the best stoichiometry to use. Thus provided good interatomic potentials are available, it is possible to predict some of the experimental conditions that should be used for the production of the best quality thin films. For sputtering simulations, we were able to show how the surface topography first develops and then stabilises and how fcc metals retain their crystalline form during the sputtering process. The method is computationally feasible because a fast algorithm for saddle point searches which are conducted in parallel means that energy barriers for diffusion can be calculated on-the-fly. The method is, in principle, applicable to many different atomistic phenomena where dose effects are important so should be especially useful in the study of radiation damage in reactor vessels and radioactive decay of waste forms. It should also have many other applications in the study of chemical reactions and biological systems.

${ }^{1}$ B. J. Alder and T. E. Wainwright, J. Chem. Phys. 27, 1208 (1957).

${ }^{2}$ J. B. Gibson, A. N. Goland, M. Milgrim, and G. H. Vineyard, Phys. Rev. 120, 1299 (1960).
${ }^{3}$ E. McGee, R. Smith, and S. D. Kenny, Int. J. Mater Res. 98, 430 (2007).

${ }^{4}$ L. Verlet, Phys. Rev. 159, 98 (1967).

${ }^{5}$ A. F. Voter, F. Montalenti, and T. C. Germann, Annu. Rev. Mater. Res. 32, 321 (2002).

${ }^{6}$ M. R. Sørensen and A. F. Voter, J. Chem. Phys. 112, 9599 (2000).

${ }^{7}$ A. F. Voter, Phys. Rev. Lett. 78, 3908 (1997).

${ }^{8}$ A. Laio and M. Parrinello, Proc. Natl. Acad. Sci. U.S.A. 99, 12562 (2002).

${ }^{9}$ A. K. Faradjian and R. Elber, J. Chem. Phys. 120, 10880 (2004).

${ }^{10}$ G. Henkelman and H. Jønsson, J. Chem. Phys. 111, 7010 (1999).

${ }^{11}$ K. A. Fichthorn and R. A. Miron, Springer Proc. Phys. 123, 7 (2009).

${ }^{12}$ A. F. Voter, "Radiation effects in solids," in Introduction to the Kinetic Monte Carlo Method (Springer-Verlag, Berlin, 2005).

${ }^{13}$ S. Numazawa, M. Ranjan, K. H. Heinig, S. Facsko, and R. Smith, J. Phys. Condens. Matter 23, 222203 (2011).

${ }^{14}$ M. G. Evans and M. Polanyi, Trans. Faraday Soc. 31, 875 (1935).

${ }^{15}$ H. Eyring, J. Chem. Phys. 3, 107 (1935).

${ }^{16}$ G. Henkelman and H. Jønsson, Chem. Phys. 115, 9657 (2001).

${ }^{17}$ J. A. Sprague, F. Montalenti, B. P. Uberuaga, J. D. Kress, and A. F. Voter, Phys. Rev. B 66, 205415 (2002).

${ }^{18}$ D. E. Harrison, N. S. Levy, J. P. Johnson, and H. M. Effron, J. Appl. Phys. 39, 3742 (1968).

${ }^{19}$ G. K. Wehner, J. Appl. Phys. 26, 1056 (1955).

${ }^{20}$ L. Kittiratanawasin and R. Smith, Nucl. Instrum. Methods Phys. Res. B 269, 1712 (2011).

${ }^{21}$ H. Berendsen, J. Postma, and W. Van Gunderstan, J. Chem. Phys. 81, 3684 (1984).

${ }^{22}$ A. Hallil, R. Tetot, F. Berthier, I. Braems, and J. Creuze, Phys. Rev. B 73, 165406 (2006).

${ }^{23}$ L. J. Vernon, S. D. Kenny, R. Smith, and E. J. Sanville, Phys. Rev. B 83, 075412 (2011).

${ }^{24}$ G. J. Ackland, G. Tichy, V. Vitek, and M. W. Finnis, Philos. Mag. A 56, 735 (1987).

${ }^{25}$ J. F. Ziegler, J. P. Biersack, and U. Littmark, The Stopping and Range of Ions in Matter (Pergamon, New York, 1985).

${ }^{26}$ N. W. Ashcroft and N. D. Mermin, Solid State Physics (Holt, Rinehart and Winston, New York, 1976).

${ }^{27}$ A. F. Voter, in Radiation Effects in Solids, NATO Science Series II: Mathematics, Physics and Chemistry, Vol. 235, edited by K. Sickafus, E. A. Kotomin, and B. P. Uberuaga (Springer, Berlin, 2007), p. 1.

${ }^{28}$ L. J. Vernon, Ph.D. dissertation, Loughborough University, 2010.

${ }^{29}$ G. Henkelman, B. P. Uberuaga, and H. Jønsson, J. Chem. Phys. 113, 9901 (2000).

${ }^{30}$ G. H. Vineyard, J. Phys. Chem. Solids 3, 121 (1957).

${ }^{31}$ S. Yastrebov and R. Smith, Nucl. Instrum. Methods Phys. Res. B 80, 145 (2001).

${ }^{32}$ C. J. Howe, M. D. Cropper, R. M. Wardle, P. Bailey, and T. C. Q. Noakes, Surf. Sci. 604, 1658 (2010).

${ }^{33}$ Z. H. Liu, N. M. D. Brown, and A. M. McKinley, J. Phys. Condens. Matter 9, 59 (1997)

${ }^{34}$ R. Smith and W. Möller, in Materials Research Society Symposium Proceedings, Boston, November 2010 (Cambridge University Press, Cambridge, England, 2011), Vol. 1298, p. 191.

${ }^{35}$ S. Wendt, P. Sprunger, E. Lira, G. Madsen, Z. Li, J. Hanson, J. Matthiesen, A. Blekinge-Rasmussen, E. Laegsgaard, B. Hammer, and F. Besenbacher, Science 320, 1755 (2008).

${ }^{36}$ E. J. Sanville, L. J. Vernon, S. D. Kenny, R. Smith, Y. Moghaddam, C. Browne, and P. Mulheran, Phys. Rev. B 80, 235308 (2009).

${ }^{37}$ P. Stone, R. A. Bennett, and M. Bowker, New J. Phys. 1, 8 (1999).

${ }^{38} \mathrm{R}$. Behrisch, Sputtering by Particle Bombardment I (Springer-Verlag, Berlin, 1981).

${ }^{39}$ B. J. Garrison, N. Winograd, D. M. Deavon, C. T. Reimann, D. Y. Lo, T. A. Tombrello, D. E. Harrison, and M. H. Shapiro, Phys. Rev. B 37, 7197 (1988). 